\title{
MODELO DE IMPACTO ECONÓMICO PARA LAS SITUACIONES DE RACHAS MÁXIMAS EXTREMAS EN LA ESPAÑA PENINSULAR
}

\author{
Macarena RODRIGO FERNÁNDEZ ${ }^{1,2}$, José Antonio LÓPEZ DÍAZ² \\ ${ }^{I}$ Consorcio de Compensación de Seguros (CCS). Madrid. \\ ${ }^{2}$ Agencia Estatal de Meteorología (AEMET). Madrid. \\ ccseguros@aemet.es, jlopezd@aemet.es
}

\section{RESUMEN}

En este trabajo se presenta el modelo de impacto económico para las situaciones de rachas máximas extremas en la España peninsular. Este modelo tiene como objetivo fundamental proporcionar información significativa para la cobertura del riesgo, y su correspondiente evaluación, para cada situación de Tempestad Ciclónica Atípica (TCA) y para un corto período de tiempo.

El modelo está basado en la aplicación conjunta de una climatología y un análisis de las pérdidas de las situaciones pasadas de TCA. Su uso tendrá en cuenta las características de la TCA y la valoración catastral total de los municipios afectados por viento extremo.

Los resultados iniciales muestran estimaciones iniciales de las pérdidas esperadas sólo para algunos municipios. Posteriormente se extienden estos resultados para la totalidad del área que comprende la España peninsular de manera que se consiga tener un campo suavizado. Los resultados se presentan a nivel de municipios por ser la unidad aplicada por el CCS en la cobertura de daños.

Mediante este modelo el CCS, que proporciona seguros contra las situaciones de rachas máximas extremas y compensa los daños producidos, dispondrá de manera rápida y eficiente de una información valiosa frente a futuras situaciones de TCA.

Palabras clave: tempestad ciclónica atípica, racha máxima, riesgo extraordinario, impacto económico, seguros.

\section{ABSTRACT}

In this work we present the economic impact model of extreme wind gust situations in peninsular Spain. The main aim of this model is to provide significant information for covering risk and corresponding evaluation for extreme wind situations (TCA) in a short period of time.

The model is based on the joint implementation of a climatology and an analysis of covered losses of past situations. It takes into account the characteristics of the TCA and the cadastre total evaluation for the area affected by extreme wind, at municipality level.

The preliminary results provides initial estimates of the cost of expected losses only for some municipalities. Afterwards, these results are extended to the entire area 
of peninsular Spain achieving a smooth field. The results are shown at municipality level because this is the unit applied by the CCS in damage coverage.

Using this model the CCS, which provides insurance against extreme wind gust situations and compensates damage, determines quickly and efficiently valuable information against future situations of TCA.

Key words: atypical cyclonic storm, maximum gust, extraordinary risk, economic impact, insurance.

\section{INTRODUCCIÓN}

El Consorcio de Compensación de Seguros, CCS, es el organismo nacional que cubre los daños producidos por ciertos fenómenos meteorológicos extremos. Uno de estos fenómenos es la Tempestad Ciclónica Atípica o TCA en el que están incluidos los vientos extraordinarios, rachas máximas de viento superiores a $120 \mathrm{~km} / \mathrm{h}$. Se entenderá por racha el mayor valor de la velocidad del viento, sostenida durante un intervalo de tres segundos. La forma establecida en la que el CCS indemniza, en régimen de compensación, las pérdidas derivadas de acontecimientos extraordinarios acaecidos en España y que afecten a riesgos en ella situados, está fijada por el Reglamento de Seguros de Riesgos Extraordinarios, RSRE.

Con objeto de la delimitación geográfica del área de afectación del fenómeno meteorológico descrito, el Consorcio de Compensación de Seguros facilitará a la Agencia Estatal de Meteorología, AEMET, cuantas mediciones ajenas a la misma reciba o pueda recabar, a efectos de su contraste por la Agencia, y solicitará su colaboración en la delimitación geográfica mediante la extrapolación, con los criterios científicos más avanzados, de las mediciones existentes, de forma que se procure la mayor homogeneidad posible en la definición del área y se evite la exclusión de puntos aislados respecto de los que exista duda razonable, incluso aunque pudieran carecer de mediciones específicas, teniendo en consideración las registradas en los municipios limítrofes y, en su caso, los colindantes con éstos (Consorcio de Compensación de Seguros, 2016, p.38).

En la España peninsular se presentan considerables dificultades para delimitar las áreas en las que se cumple la condición de TCA debidas, principalmente, a la falta de observaciones y la complejidad del terreno. AEMET, que realiza los informes técnicos para la delimitar estas zonas, aplica la técnica de interpolación geoestadística del krigeado universal que se apoya en los datos de observación y toma como variables externas la elevación del terreno, la distancia a la costa y las salidas de racha máxima de alcance más corto del modelo operativo de predicción HIRLAM 0.05 (High Resolution Limited Area Model), (Rodrigo y López, 2013).

El krigeado estima en un punto cualquiera el valor de racha máxima como una combinación lineal de las observaciones en el resto de puntos debidamente ponderadas (se busca que el estimador sea insesgado y que tenga varianza mínima). Esta técnica del krigeado universal, utilizada para estimar las áreas de rachas máximas de viento, se basa en un modelo de tendencia lineal en el que entran en juego las variables auxiliares ya citadas obteniendo, de esta manera, variaciones suaves en la interpolación (Burrough y McDonnell, 1998). 
Además, la estructura del semivariograma se especifica "sin efecto pepita", es decir, el krigeado es exacto: en los puntos de observación el valor estimado coincide con el valor observado, (Cressie, 1991). Los datos observados son los que tienen mayor fuerza legal por lo que es indispensable respetar al máximo estos valores tal y como lo hace el método empleado.

En este trabajo se presenta el modelo de impacto económico para las situaciones de rachas máximas extremas en la España peninsular. Este modelo tiene como objetivo fundamental proporcionar información significativa para la cobertura del riesgo, y su correspondiente evaluación, para cada situación de TCA y para un corto período de tiempo.

\section{MÉTODOS}

\subsection{Modelo Preliminar}

El Modelo Preliminar se desarrolla con la finalidad de obtener unas estimaciones iniciales del coste de las pérdidas esperadas por vientos extremos a nivel de municipios. Para ello se genera una climatología de las zonas afectadas por viento extraordinario en el período de años comprendido entre 2009 y 2014, ambos incluidos, y por otro lado, se realiza un análisis de las pérdidas cubiertas (costes actualizados) en situaciones reales para estos mismos años utilizando datos aportados por el CCS. La aplicación conjunta de esta información constituye el denominado Modelo Preliminar.

\section{1.a. Climatología}

Para la construcción de una climatología de vientos extraordinarios empleamos los datos de observación de las estaciones de AEMET desde el año 2009, fecha en la que el número de estaciones comienza a ser homogéneo.

Posteriormente estudiamos la frecuencia con la que se producen las situaciones de viento extremo incluyendo sólo aquellos días en los que al menos una estación de observación presenta rachas máximas superiores a $110 \mathrm{~km} / \mathrm{h}$. Para cada uno de estos días se realizan las estimaciones de racha máxima para el área de la España peninsular y los días que conforman esta climatología serán finalmente aquellos en los que existan valores estimados superiores a 120 km/h (Bivand, Pebesma y Gómez-Rubio, 2008).

Sin embargo, las situaciones reales de TCA suelen incluir varios días, asociadas a la situación sinóptica acaecida, para poder simular estas condiciones se aplican unos criterios restrictivos. En primer lugar unimos los días consecutivos (cronológicamente, hasta un máximo de 5 días, puesto que ninguna situación suele superar este período). Además, los días que pudieran aparecer sueltos se unen a las situaciones más cercanas (siempre que la separación sea de 1 único día). De esta manera se obtienen las situaciones de TCA que contienen nuestra climatología y para ellas se realizan de nuevo las estimaciones de racha máxima según el procedimiento operativo, utilizando el krigeado universal.

La aplicación de este procedimiento tiene en cuenta la modificación reflejada en el RSRE relativa al límite impuesto en el valor de racha máxima para las situaciones de TCA. En fecha anterior al 27 de octubre de 2011 la definición de TCA exige un umbral superior a $135 \mathrm{~km} / \mathrm{h}$ para la variable de racha máxima, frente a los $120 \mathrm{~km} / \mathrm{h}$ 
actuales. Además, en este primer período, en el que el límite es de $135 \mathrm{~km} / \mathrm{h}$, no se incorpora el modelo HIRLAM en la interpolación, ya que las estimaciones realizadas en ese momento tampoco tenían en cuenta esta información.

\section{1.b. Análisis de las pérdidas}

Para el estudio de la distribución espacial de las pérdidas se aplican los datos aportados por el CCS de las pérdidas cubiertas (costes actualizados) en situaciones reales.

Inicialmente se estima la pérdida para una TCA típica tomando todo el período en estudio. Se realiza un sumatorio sobre los costes reales y se divide por el número de TCAs dadas en nuestra climatología, a nivel de municipios, ecuación 1.

$$
\text { Pérdida }\left.\right|_{\text {muni }}(€)=\frac{\sum_{i \in \text { muni }} \cos \operatorname{tes}^{i}(C C S)}{\sum_{i \in \text { muni }} n^{\circ} T C A^{i}(\text { CLIMA) }}
$$

(Ecuación 1)

Un desarrollo posterior en el modelo lleva a realizar este mismo cálculo para cada municipio y para cada mes de manera que si en alguno de ellos hay coste asociado sin TCA en la climatología se le imputa el valor de 1 (como si se hubiese dado). Esta modificación en el modelo subsana el error de perder información aportada por el CCS debido a la falta de sincronización entre las fechas de las situaciones reales y las estimadas en la climatología.

\subsection{Evolución del Modelo Preliminar}

Las pérdidas medias calculadas hasta el momento están referenciadas para diferentes períodos, según el límite impuesto de racha máxima, el objetivo siguiente es referenciar estos valores al período de $120 \mathrm{~km} / \mathrm{h}$, puesto que es el límite impuesto actualmente en el RSRE. Para ello se busca la relación existente entre los municipios comunes a ambos períodos. En vista a los resultados y a la disparidad de los datos, se decide tomar la relación logarítmica. De esta manera las pérdidas medias calculadas para el período de $120 \mathrm{~km} / \mathrm{h}$ son datos directos del modelo desarrollado anteriormente, mientras que los del período de $135 \mathrm{~km} / \mathrm{h}$ son valores ajustados según una relación logarítmica.

El problema que se presenta es que los valores obtenidos se presentan sólo para ciertos municipios, aquellos de los que se disponía información. Para poder obtener una estimación de la pérdida media para todos los municipios de la España peninsular, creando un campo suavizado, se efectúa una regresión múltiple del logaritmo de la pérdida media (en miles de euros) por cada 1000 personas sobre otras variables relevantes adicionales tras un proceso de selección paso a paso de las más significativas. La inclusión de estas variables surge de la idea de que la naturaleza catastrófica de un fenómeno depende de diversos factores, no sólo del valor extremo que presente el elemento climático, sino de la distribución de la población o de las características geomorfológicas, entre otros muchos (García-Legaz y Valero, 2003). En concreto para nuestro modelo se han utilizado: logaritmo decimal de la población, desviación estándar de la altitud, distancia al mar media y logaritmo decimal de la extensión (número de puntos de grid). 


\subsection{Modelo Físico y Modelo Actuarial}

El objetivo es construir un modelo categórico final de manera que se evalúe de forma rápida el impacto económico previsto para cada municipio en cada nueva TCA. Así, teniendo en cuenta el modelo preliminar desarrollado, se procede a construir el Modelo de Impacto Económico que consta de dos modelos claramente diferenciados: Modelo Físico y Modelo Actuarial.

\section{3.a. Modelo Físico}

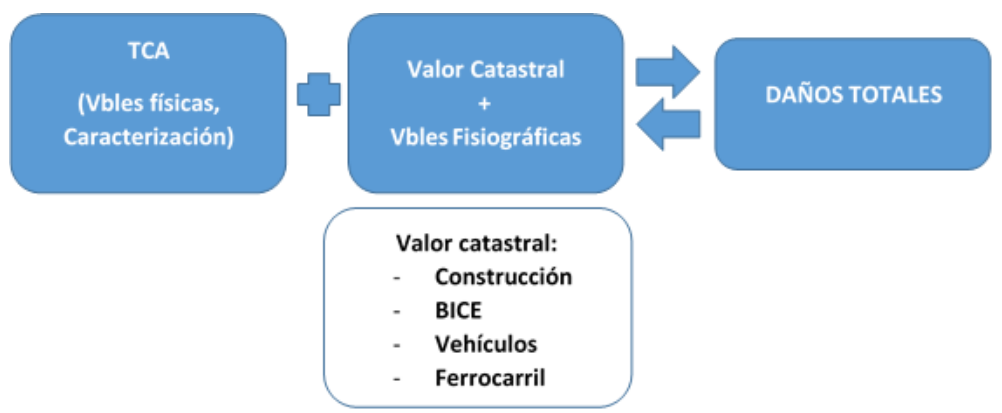

Fig. 1: Esquema general del Modelo Físico.

La construcción del modelo físico tiene en cuenta que los daños totales producidos en una determinada TCA dependen de la propia situación, estimada y caracterizada mediante variables físicas, y de la valoración catastral presente en las zonas afectadas, e incluso de ciertas variables fisiográficas. El esquema general de este modelo se presenta en la figura 1, y consiste en estudiar situaciones pasadas que permitan relacionar el comportamiento que presentan las situaciones de TCA, los valores catastrales de las zonas afectadas y los daños totales producidos.

Puesto que las situaciones con vientos extraordinarios presentan características y extensiones muy diferentes surge la necesidad de caracterizar estos eventos. Para ello se han definido tres índices de caracterización que son calculados para cada municipio: índice máximo (ecuación 2), índice de excesos (ecuación 3) e índice de excesos cuadrático (ecuación 4). Para los índices de excesos se utiliza el umbral de $90 \mathrm{~km} / \mathrm{h}$ puesto que suele ser el límite impuesto por las compañías de seguros para aplicar la cobertura de los daños producidos.

$$
\begin{gathered}
\left.I_{\max }\right|_{\text {muni }}=\max _{i \in \text { muni }}\left(R_{\max }^{i}\right) \\
\left.I_{\text {exc }}\right|_{\text {muni }}=\frac{\sum_{i \in \text { muni }} \max \left(0, R_{\max }^{i}-90\right)}{n^{\circ} \text { ptos grid }\left.\right|_{\text {muni }}}
\end{gathered}
$$

(Ecuación 2)

(Ecuación 3) 


$$
\left.I_{\text {exc } 2}\right|_{\text {muni }}=\frac{\sum_{i \in \text { muni }} \max \left(0, R_{\max }^{i}-90\right)^{2}}{n^{\circ} \text { ptos } \text { grid }\left.\right|_{\text {muni }}}
$$

(Ecuación 4)

siendo $R_{\max }^{i}$ la racha máxima en el punto de grid i-ésimo.

Además, se añaden otros tres índices definidos del mismo modo pero tomando la variable de velocidad máxima del viento medida en 10 minutos, esta variable se considera de importancia para la caracterización de las situaciones en términos de su impacto en daños. Para los índices de excesos, en este caso, se impone el umbral de $50 \mathrm{~km} / \mathrm{h}$ puesto que es el valor correspondiente a los $90 \mathrm{~km} / \mathrm{h}$ de la velocidad de racha máxima según un estudio climatológico en términos de frecuencias.

Tal y como se muestra en el esquema de la figura 1, la construcción de este modelo tiene en cuenta diferentes variables fisiográficas, al igual que el Modelo Preliminar, y ciertos valores relativos al catastro, que sustituirán a la variable población empleada en la regresión múltiple del modelo citado.

Los valores catastrales incluidos para cada municipio se refieren, principalmente, a los valores catastrales de construcción (tipo urbano y rústico) así como los valores catastrales tipo BICE (Bienes Inmuebles de Características Especiales) que son obtenidos a través de la Dirección General del Catastro, estos son de aplicación en todo el territorio nacional exceptuando los casos especiales de País Vasco y Navarra. Sin embargo, se realiza un estudio para estos casos especiales, de manera que podamos tener una estimación a nivel municipal para estas provincias, (R Core Team, 2015). Por un lado, para el tipo urbano, se aplica un factor de conversión que tiene en cuenta los valores de construcción y de suelo del resto de provincias y que presenta una relación significativa con la densidad de población. Para el caso rústico, aplicamos una recta de regresión elaborada con los valores para el resto de provincias. Ambos estudios permiten elaborar unas estimaciones de los valores catastrales de construcción para los municipios pertenecientes a los casos especiales de País Vasco y Navarra.

Se añade información adicional relativa, por un lado, a los vehículos, tomando sus valores para cada término municipal y desglosados por tipo, información recogida de la Dirección General de Tráfico, y por otro lado la información relativa a la red ferroviaria, donde se incluyen estimaciones elaboradas por el CCS del valor de la exposición de la red ferroviaria de Adif para cada término municipal.

La construcción de este modelo permitirá aplicarlo del mismo modo, en sentido inverso, de manera que podamos obtener inmediatamente los daños totales producidos en una determinada situación de TCA, aplicando los valores catastrales, en un corto plazo de tiempo.

\section{3.b. Modelo Actuarial}

La construcción de este modelo se presenta en la figura 2 a modo de esquema. Para ello es importante diferenciar los conceptos de indemnización y daño total. La indemnización es la principal obligación del asegurador, motivada por la concurrencia de un siniestro indemnizable y notificado por el asegurado y determinada por la tasación de los daños producidos por el siniestro. Los valores de los daños totales se obtienen una vez aplicado los datos de franquicia, infraseguro y penetración. 


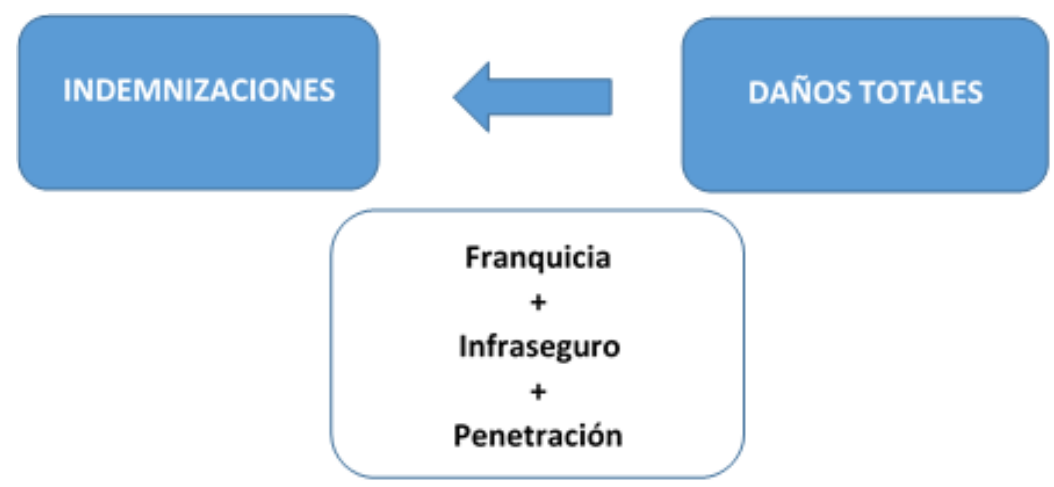

Fig. 2: Esquema general del Modelo Actuarial.

Definimos a continuación los conceptos aplicados para este modelo. Todas estas definiciones proceden del diccionario de MAPFRE de seguros, Mutua de la Agrupación de Propietarios de Fincas Rústicas de España. La franquicia es la cantidad por la que el asegurado es propio asegurador de sus riesgos y en virtud de la cual, en caso de siniestro, soportará con su patrimonio la parte de los daños que le corresponda. El infraseguro es la situación que se origina cuando el valor que el asegurado o contratante atribuye al objeto garantizado en una póliza es inferior al que realmente tiene. La penetración es la participación de la actividad aseguradora en la economía de un país o región. Estos últimos valores son recogidos a partir del documento de UNESPA, Asociación Empresarial del Seguro, Memoria Social UN14, de la tabla 1: "Tasas de penetración de distintos tipos de seguro, por Comunidades Autónomas".

El modelo actuarial se aplica en el sentido en el que obtenemos las indemnizaciones ya que el resto de valores (daños totales, franquicia e infraseguro) son facilitados por el CCS, a excepción de los valores de penetración que, como ya hemos mencionado, se recogen del documento de UNESPA.

\section{RESULTADOS}

En primer lugar, el estudio climatológico tiene en cuenta la necesaria homogeneidad que tendrá que existir en el número de estaciones de observación anual. En la figura 3 se muestra el período comprendido entre el año 2000 a 2014 donde se comprueba que el número de estaciones de AEMET comienza a ser homogéneo en el año 2009, fecha de la que parte nuestro estudio.

La caracterización de las situaciones de TCA también se realiza en el estudio climatológico tal y como muestra la figura 4. En este análisis simplemente se han diferenciado dos tipos de TCA: aquellas que denominamos normales, que engloban aquellas que se dan con más frecuencia y sus extensiones son medias o pequeñas, frente a las denominadas grandes, que suelen darse con menos frecuencia (en la figura número en rojo) y son de extensiones mucho mayores. El criterio tomado para diferenciar estos dos tipos toma el número de puntos de grid que superan los $80 \mathrm{~km} / \mathrm{h}$ para los valores estimados de racha máxima, el límite entre 120000 puntos de grid separa ambos patrones de TCA. 
Estaciones de observación AEMET (2000-2014)

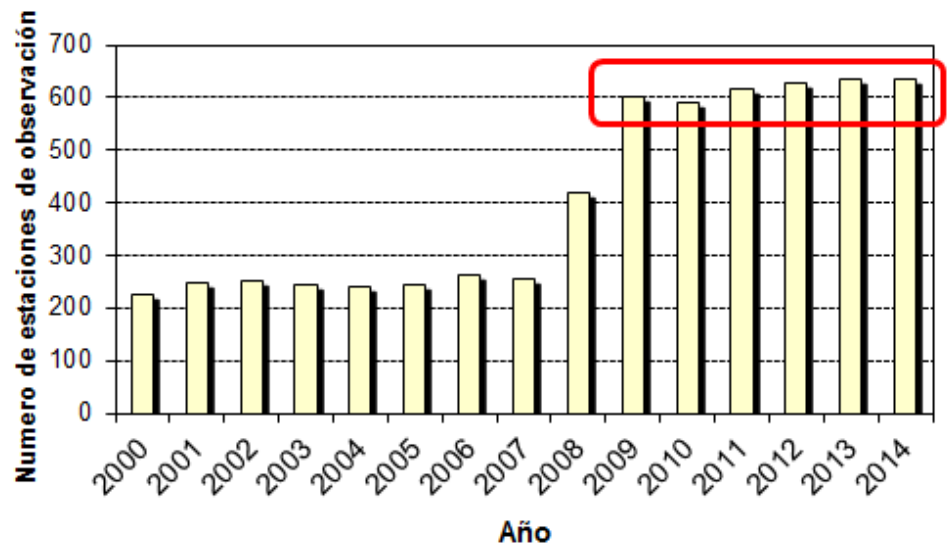

Fig. 3: Número de estaciones de observación AEMET (racha máxima). 2000 a 2014.

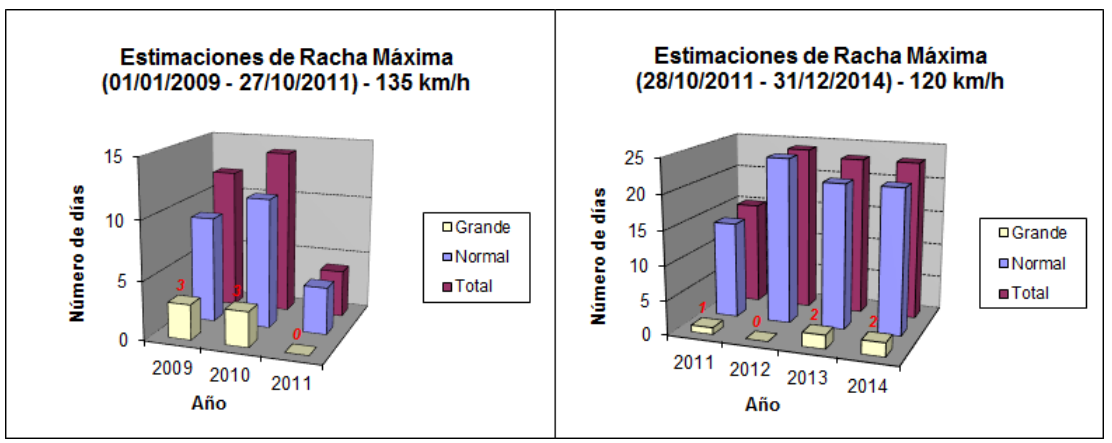

Fig. 4: Caracterización de las situaciones de TCA de la climatología.

En la figura 5 se muestran los resultados obtenidos para el Modelo Preliminar. En el mapa de la izquierda se muestran los valores de la pérdida media (en miles de euros) para cada municipio y para cada TCA. Estos valores se obtienen una vez realizada la transformación logarítmica para el período de referencia de $120 \mathrm{~km} / \mathrm{h}$ y para los municipios de los que se dispone información inicialmente. En el mapa de la derecha se muestra el resultado extrapolado a toda el área de la España peninsular obtenido por la aplicación de una regresión múltiple. En este caso los valores indican la pérdida media (en miles de euros) por persona, para cada municipio y para cada TCA.

El planteamiento final para la construcción del Modelo de Impacto Económico incluye información referida al catastro puesto que estos valores presentan una fuerte dependencia con la pérdida media que supone cualquier situación de TCA.

El estudio para obtener estos valores ha sido bastante laborioso y en este trabajo sólo mostraremos los resultados referentes a los valores catastrales de construcción (tanto para tipo urbano como para tipo rústico), especialmente aquellos que se refieren a la obtención de dicha información para los casos especiales de País Vasco y Navarra. 


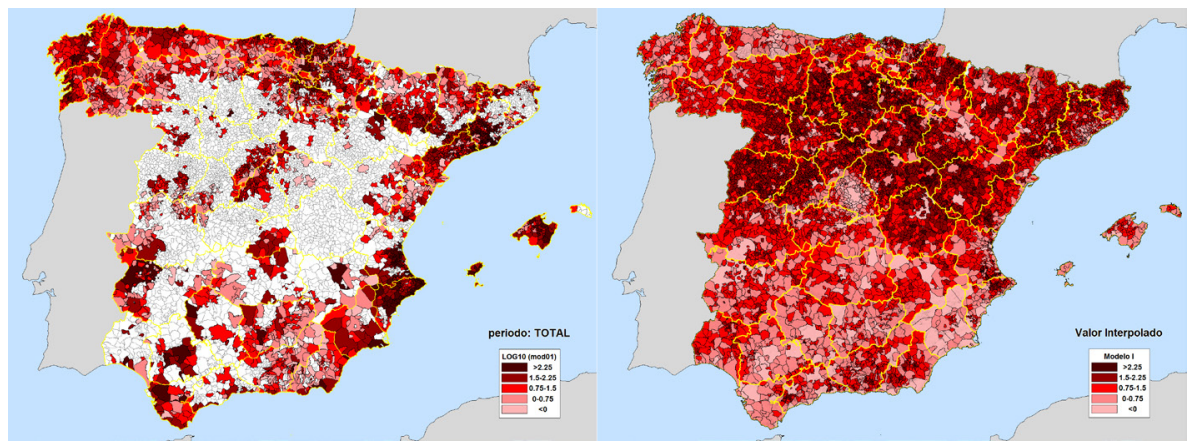

Fig. 5: Resultados del Modelo Preliminar.

Para el tipo urbano se aplica un factor de conversión, considerado constante para cada municipio perteneciente a la misma provincia. Este factor, calculado para cada provincia, es la razón entre el valor de la construcción y el valor del suelo. Posteriormente se realiza un estudio entre este factor de conversión y las variables de renta per cápita y densidad de población para cada una de las provincias. Se comprueba que tenemos una regresión significativa para la población y sin embargo no se aprecia dependencia con la recta per cápita. En la figura 6 se muestran los pares de puntos referentes al cociente entre el valor de construcción y el valor del suelo frente a la densidad de población para cada una de las provincias. Se han incluido los valores obtenidos para las provincias de los casos especiales que nos han permitido calcular sus valores catastrales de la construcción.

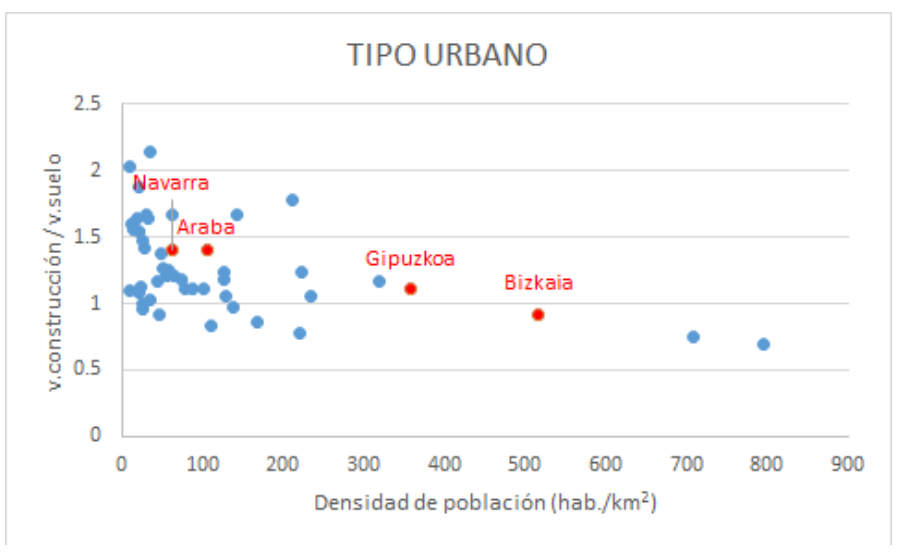

Fig. 6: Relación entre el factor de conversión y la densidad de población para tipo urbano.

Para el caso rústico se comparan los pares de valores que tenemos disponibles para el resto de provincias, valor catastral de construcción frente al valor catastral total, ver figura 7. La correlación que se presenta es buena y tomamos la regresión lineal como válida, esta recta, forzada a pasar por el punto $(0,0)$, nos permite sacar los valores de construcción para los municipios pertenecientes al País Vasco y Navarra. 


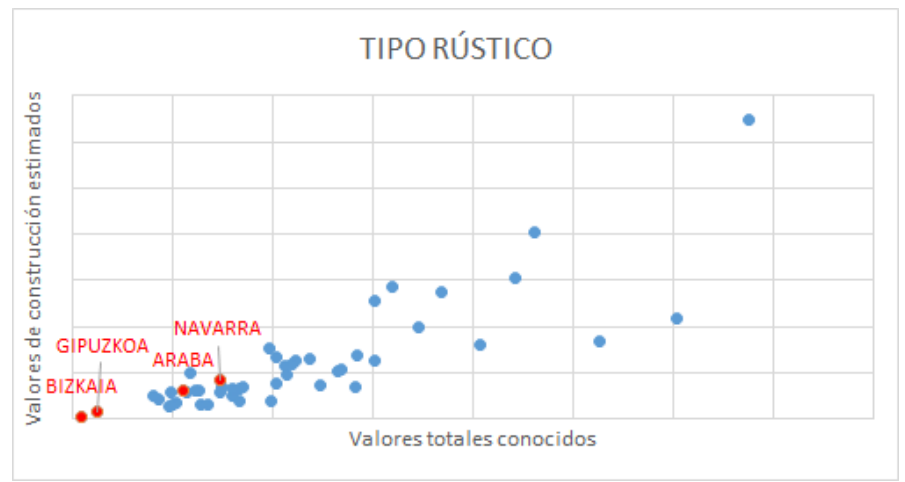

Fig. 7: Relación entre el valor de construcción y el valor del suelo para tipo rústico.

\section{DISCUSIÓN}

Hemos construido un Modelo Preliminar basado, inicialmente, en una climatología de situaciones de rachas máximas extremas y el inventario de las indemnizaciones realizadas por el CCS de TCAs pasadas. Este modelo relaciona los dos períodos en que el límite de racha máxima impuesto en el RSRE es diferente, dando lugar a un modelo logarítmico. Además, para extender la estimación de pérdida a todos los municipios y suavizar sus valores se aplica una regresión múltiple en la que entran en juego otras variables relevantes.

El objetivo final consiste en obtener un modelo categórico que permita evaluar de forma rápida el impacto económico previsto para cada municipio ante una nueva situación de TCA. Esto requiere caracterizar cada una de las situaciones de TCA en términos de su previsible impacto. Para ello se han desarrollado una serie de índices que se consideran suficientemente representativos. La construcción del modelo incluye, por último, información referente al catastro, concretamente se introducen los valores catastrales de construcción (tipo urbano, rústico y de Bienes Inmuebles de Características Especiales), valor de la exposición de la red ferroviaria y de los vehículos. Finalmente el modelo permitirá obtener los daños totales a partir de esta información.

Una ampliación del modelo estudiará la posibilidad de dar los valores de daños totales en términos de indemnizaciones. Para ello se empleará información relativa a franquicia, infraseguro y penetración.

\section{AGRADECIMIENTOS}

Este trabajo surge gracias a la colaboración entre la Agencia Estatal de Meteorología y el Consorcio de Compensación de Seguros los cuales, junto a AT Sistemas, patrocinan el proyecto. Los autores desean agradecer el apoyo de muchas personas que pertenecen a estas organizaciones y que hacen posible estas investigaciones.

\section{REFERENCIAS}

Bivand, R.S., Pebesma, E.J. y Gómez-Rubio, V. (2008). Applied Spatial Data Analysis with R. Editorial Springer. 
Burrough, P.A. y McDonnell, R.A. (1998). Principles of Geographical Information Systems. Editorial Oxford University Press.

Consorcio de Compensación de Seguros. (2016). Recopilación Legislativa. Enero 2016. Recuperado de: http://www.consorseguros.es/web/documents/10184/44193/Reco pilacion legislativa 2016/1fa097fb-3407-451b-be59-fcf6adc066d1.

Cressie, N. (1991). Statistics for spatial data. Editorial: A Wiley - Interscience Publication.

García-Legaz Martínez, C. y Valero Rodríguez, F. (2003). Riesgos Climáticos e Impacto Ambiental. Madrid. Editorial Complutense.

R Core Team (2015). R: A language and environment for statistical computing. R Foundation for Statistical Computing, Vienna, Austria. https://www.R-project.org/.

Rodrigo, M. y López, J.A. (2013). Improving the AEMET operational procedure for estimating areas of maximum wind gusts, vol. 10, 35-44. Retrieved from: http:// www.tethys.cat/sites/default/files/pdf/articles/10tethys-04-eng.pdf. 